\title{
Numerical solution for tachyon vacuum in the Schnabl gauge
}

\author{
E. Aldo Arroyo ${ }^{a}$ and Matěj Kudrna ${ }^{b}$ \\ ${ }^{a}$ Centro de Ciências Naturais e Humanas, Universidade Federal do ABC, \\ Santo André, 09210-170 São Paulo, SP, Brazil \\ ${ }^{b}$ Institute of Physics of the ASCR, v.v.i., \\ Na Slovance 2, 18221 Prague 8, Czech Republic \\ E-mail: aldo.arroyo@ufabc.edu.br, kudrnam@fzu.cz
}

ABSTRACT: Based on the level truncation scheme, we develop a new numerical method to evaluate the tachyon vacuum solution in the Schnabl gauge up to level $L=24$. We confirm the prediction that the energy associated to this numerical solution has a local minimum at level $L=12$. Extrapolating the energy data of $L \leq 24$ to infinite level, we observe that the energy goes towards the analytical value -1 , nevertheless the precision of the extrapolation is lower than in the Siegel gauge. Furthermore, we analyze the Ellwood invariant and show that its value converges monotonically towards the expected analytical result. We also study the tachyon vacuum expectation value (vev) and some other coefficients of the solution. Finally, some consistency checks of the solution are performed, and we briefly discuss the search for other Schnabl gauge numerical solutions.

KeYwords: String Field Theory, Tachyon Condensation

ArXiv EPrint: 1908.05330 


\section{Contents}

1 Introduction 1

2 Solving the equations of motion in the Schnabl gauge 3

3 Coefficients of the tachyon condensate $r$

4 The vacuum energy and the gauge invariant overlap 11

5 Checking some symmetries $\quad 16$

6 Out-of-gauge equations of motion $\quad 18$

$\begin{array}{lll}7 & \text { Summary and discussion } & 19\end{array}$

$\begin{array}{ll}\text { A Extrapolations to infinite level } & 21\end{array}$

B Search for other solutions $\quad 24$

\section{Introduction}

In the context of Witten's open bosonic string field theory [1], using a solution for tachyon condensation in the Schnabl gauge [2], the first analytic proof of Sen's first conjecture [3, 4] has been performed. Based on Schnabl's work, many analytical and numerical tools have been developed [5-21] and the techniques have been employed in the construction and analysis of new analytical solutions [22-33].

In the construction of this analytical solution for tachyon condensation, the Schnabl gauge condition, $\mathcal{B}_{0} \Psi=0$, plays a fundamental role. As shown in reference [2], thanks to the combination of the $\mathcal{B}_{0}$ gauge with the $\mathcal{L}_{0}$ level truncation in certain sectors of the state space formed by the $\tilde{c}_{n}$ modes, $\mathcal{L}_{0}+\mathcal{L}_{0}^{\dagger}$ and $\mathcal{B}_{0}+\mathcal{B}_{0}^{\dagger}$ operators acting on the vacuum, the entire set of equations of motion $Q \Psi+\Psi * \Psi=0$ can be solved exactly, in a recursive way. The result of such a calculation gives us the analytical solution $\Psi$, which, in terms of wedge states with ghost insertions, can be written as

$$
\begin{aligned}
\Psi & =\lim _{N \rightarrow \infty}\left[\psi_{N}-\sum_{n=0}^{N} \partial_{n} \psi_{n}\right], \\
\psi_{n} & =\frac{2}{\pi^{2}} U_{n+2}^{\dagger} U_{n+2}\left[\left(\mathcal{B}_{0}+\mathcal{B}_{0}^{\dagger}\right) \tilde{c}\left(-\frac{\pi}{4} n\right) \tilde{c}\left(\frac{\pi}{4} n\right)+\frac{\pi}{2}\left(\tilde{c}\left(-\frac{\pi}{4} n\right)+\tilde{c}\left(\frac{\pi}{4} n\right)\right)\right]|0\rangle,
\end{aligned}
$$

where $\psi_{N}$, with $N \rightarrow \infty$, is called the phantom term $[2,5,12,13]$. The equations (1.1) and (1.2) can be used to expand the analytical solution in the state space of Virasoro $L_{0}$ 


\begin{tabular}{|c|c|c|}
\hline$L$ & $c_{1}|0\rangle$ & $E^{\mathrm{Sch}}$ \\
\hline 0 & 0.456177990470 & -0.684616159915 \\
\hline 2 & 0.544204232320 & -0.959376599521 \\
\hline 4 & 0.548938521247 & -0.994651904750 \\
\hline 6 & 0.548315148955 & -1.003983765388 \\
\hline 8 & 0.547321883647 & -1.007110280219 \\
\hline 10 & 0.546508411314 & -1.008189759705 \\
\hline
\end{tabular}

Table 1. $(L, 3 L)$ level truncation results of reference [36] for the tachyon vev and vacuum energy in the Schnabl gauge up to level $L=10$.

eigenstates. This level expansion of the solution is very useful for the numerical evaluation of the energy. It is important to mention that one slight disadvantage of the $\mathcal{B}_{0}$ gauge is that the gauge fixing condition is broken by the Virasoro $L_{0}$ level truncation. In reference [2], the author conjectured that the level dependent Schnabl gauge fixing condition would not pose problems and using the high $L_{0}$ level truncation computations of Moeller and Taylor [34] and Gaiotto and Rastelli [35], it should be possible to construct a numerical solution that would converge to his analytical solution when the level goes to infinity.

The first attempt to obtain a numerical solution for tachyon vacuum in the Schnabl gauge was made by Arroyo et al. [36], using the traditional level truncation computations up to level $L=10$. By extrapolating the energy data of levels $L \leq 10$, shown in table 1 , to estimate the energy for $L>10$, the authors predicted that the energy reaches a local minimum value at level $L=12$, to subsequently turn back to approach -1 asymptotically as $L \rightarrow \infty$. Although the value of the energy for this numerical solution appears to converge to the expected analytical result, the issue whether this solution could be identified with the Schnabl analytical solution [2] when $L \rightarrow \infty$ was inconclusive. For instance, as shown in table 1 , the tachyon vev (starting at level $L=4$ ) appears to decrease with the level and it does not appear to converge to the expected analytical value $t=0.55346558$. Extrapolating the data of the tachyon vev 1 , it was predicted that the tachyon vev reaches a local minimum value at a level close to $L=26$, to then turn back to approach the expected analytical result as $L \rightarrow \infty$.

One of the main motivations of this work is to provide a conclusive evidence of the conjecture in reference [2], that the numerical solution constructed in the Schnabl gauge by means of level truncation computations can be identified with the analytical solution (1.1) when $L \rightarrow \infty$. An obvious step to accomplish this task is to perform higher level computations, this might appear as an straightforward extension of the calculations developed in reference [36].

However the numerical method used in reference [36] is not practical for levels beyond $L>12$. To see why, let us briefly explain how it works. After truncating the string field to some given level $L$, we plug this string field into the string field theory action and compute the level $(L, 3 L)$ tachyon potential. Then we impose the Schnabl gauge condition and, to obtain the numerical solution, we extremize this gauge fixed potential. Therefore this 
method needs the full $(L, 3 L)$ level truncated potential as an input, however, storing this full potential at high levels requires a huge amount of computer memory, for example, to reproduce the level 24 results in this way we would need a memory size over one petabyte.

In this work, we have managed to solve the aforementioned technical issues, and we have obtained results up to level $L=24$, using a clever numerical method based on the traditional level truncation scheme, which in principle can be applied to all general linear $b$-gauges. We have explicitly proven that the energy of the numerical solution has in fact a local minimum at level $L=12$, so the conjecture made in [36] was proven to be correct.

Regarding the prediction of the local minimum at level $L=26$ for the tachyon vev made in [36], by extrapolating the corresponding data of levels $L \leq 24$, we observe that this local minimum probably exists, but at a higher level, possibly somewhere below level $L \lesssim 42$. Although the predictions of its position are not very reliable, from the trend observed in the data up to level 24 , we are almost sure that this minimum does not happen at $L=26$ as claimed in [36].

Apart from the tachyon vev, we also analyzed the asymptotic behavior of some other coefficients of the numerical solution, and showed that they converge to the expected analytical result, although the precision is lesser than we hoped for. Furthermore, we computed the Ellwood invariant and found that its value is in agreement with the expected analytical result. By performing some consistency checks of the numerical solution, we provided an extra evidence for the conclusion that the solution can be identified with the analytical solution at the limit of infinite level.

This paper is organized as follows. In section 2, we discuss how to impose the Schnabl gauge condition (or, in general, other nontrivial linear gauge conditions) at high level numerical calculations. We implement the gauge condition using a projector acting on the full equations of motion. In section 3, we analyze the tachyon vev and some other coefficients of the numerical solution and compare them to coefficients of the analytical solution. In section 4, we present level 24 data for the tachyon vacuum energy and the gauge invariant overlap and we extrapolate these quantities to the infinite level. In section 5 , we check that the numerical solution satisfies some nontrivial identities that were discovered in [2]. In section 6 , we verify that the solution satisfies some of the out-of-gauge equations of motion. In section 7, we summarize our results and discuss some related numerical experiments. In appendix A, we provide general rules for extrapolations of various quantities to infinite level. Finally, in appendix B, we briefly mention two other numerical solutions in the Schnabl gauge.

\section{Solving the equations of motion in the Schnabl gauge}

In this section, we discuss how to adapt Newton's method, which is commonly used to solve the SFT equations numerically $[35,37]$ with nontrivial gauge conditions. More information about our numerical algorithms can be found in [38].

The string field theory action has a large amount of gauge symmetry, which is, in an infinitesimal form, given by

$$
\delta \Psi=Q \Lambda+(\Psi * \Lambda-\Lambda * \Psi) .
$$


These gauge transformations do not commute with $L_{0}^{\text {tot }}$ and therefore the gauge symmetry is broken when we truncate the action to a finite level. This may look as an advantage because the level-truncated equations of motion have only a discreet set of solutions even without any gauge fixing, but it is actually the opposite. The remnants of the gauge symmetry cause technical problems and there does not appear to be any consistent way to improve these solutions to higher levels (see [37]), which makes this approach essentially unusable. Therefore, it is necessary to make a gauge choice in the level truncation approach. We consider gauge conditions in the form of a linear constraint,

$$
\mathcal{G} \Psi=0 .
$$

Ultimately, we are interested only in the Schnabl gauge, where

$$
\mathcal{G}=\mathcal{B}_{0}=b_{0}+\sum_{k=1}^{\infty} \frac{2(-1)^{k+1}}{4 k^{2}-1} b_{2 k},
$$

but the way to solve the equations of motion does not really depend on the precise form of $\mathcal{G}$, so for now, we will work with a generic linear operator $\mathcal{G}$.

Once we impose some gauge conditions, the system of the equations of motion

$$
Q \Psi+\Psi * \Psi=0
$$

with the linear equations (2.2) becomes overdetermined and it has generically no solutions at finite level. The usual method to deal with this problem is to solve only a subset of the full equations of motion, which we write as

$$
P(Q \Psi+\Psi * \Psi)=0,
$$

where $P$ is a projector of an appropriate rank. The remaining equations

$$
(\mathbb{1}-P)(Q \Psi+\Psi * \Psi)=0
$$

are left unsolved, but, for consistent solutions, they must asymptotically approach zero as the level goes to infinity. Following [35], we call them out-of-gauge equations.

In the Siegel gauge, which is the most common choice in the level truncation approach, these issues have a very elegant solution $[34,35]$. The gauge condition

$$
b_{0}|\Psi\rangle=0
$$

can be solved simply by removing all states that contain $c_{0}$ from the spectrum. The projected equations of motion are given by derivatives of the action with respect to the remaining Siegel gauge variables, which means that the projector is simply $P=c_{0} b_{0}$.

However when we consider a more complicated gauge (which essentially means any other gauge), such simple approach no longer works and we will have to use the projector $P$ in a nontrivial way.

We usually expand the string field as

$$
|\Psi\rangle=\sum_{i} t_{i}|i\rangle
$$


where $|i\rangle$ are some basis states and $t_{i}$ is a vector of real or complex coefficients. With respect to this basis, we define the matrices

$$
\begin{aligned}
Q_{i j} & =\langle i|Q| k\rangle, \\
V_{i j k} & =\left\langle V_{3} \mid i\right\rangle|j\rangle|k\rangle,
\end{aligned}
$$

which allow us to write the level-truncated action as

$$
S(t)=-\frac{1}{g_{o}^{2}}\left(\frac{1}{2} \sum_{i, j} Q_{i j} t_{i} t_{j}+\frac{1}{3} \sum_{i, j, k} V_{i j k} t_{i} t_{j} t_{k}\right)
$$

and the equations of motion as

$$
f_{i}(t)=\sum_{j} Q_{i j} t_{j}+\sum_{j, k} V_{i j k} t_{j} t_{k}=0 .
$$

As long as there are no gauge conditions or they admit a trivial solution like in the Siegel gauge, we solve these equations using the well-known Newton's method. We start with an approximate solution $t^{(0)}$ and we iteratively improve the solution as $t^{(n+1)}=t^{(n)}+\Delta t$, where $\Delta t$ is a solution of linear equations

$$
\sum_{j} M_{i j}\left(t^{(n)}\right) \Delta t_{j}=-f_{i}\left(t^{(n)}\right)
$$

where $M_{i j}$ is the Jacobian matrix

$$
M_{i j}(t)=\frac{\partial f_{i}(t)}{\partial t_{j}}=Q_{i j}+\sum_{k}\left(V_{i j k}+V_{j i k}\right) t_{k}
$$

We repeat this procedure until we reach a desired precision of the solution.

Now consider the gauge condition (2.2). Once we expand the string fields into a basis, it transforms into a set of homogeneous linear equations

$$
\sum_{j} \mathcal{G}_{i j} t_{j}=0
$$

Using standard linear algebra, we express the matrix $\mathcal{G}$ as $^{1}$

$$
\mathcal{G}=\left(\begin{array}{cccccccc}
\overbrace{}^{1} & 0 & \ldots & 0 & a_{11} & a_{12} & \ldots & a_{1 N_{I}} \\
0 & 1 & \ldots & 0 & a_{21} & a_{22} & \ldots & a_{2 N_{I}} \\
\vdots & \vdots & \ddots & \vdots & \vdots & \vdots & \ddots & \vdots \\
0 & 0 & \ldots & 1 & a_{N_{D} 1} & a_{N_{D} 2} & \ldots & a_{N_{D} N_{I}}
\end{array}\right) .
$$

This matrix tells us how to divide the variables $t_{i}$ into $N_{I}$ independent variables $t_{i}^{(I)}$ and $N_{D}$ dependent variables $t_{i}^{(D)}$. In the Schnabl gauge, it is convenient to use the basis of $b$ and $c$ ghosts, in which the matrix $\mathcal{G}$ is quite sparse and relatively easy to work with.

\footnotetext{
${ }^{1}$ This form of the matrix is simplified for illustrative purposes. We order states by level in the actual algorithm, therefore the dependent and independent variables are not grouped together and we end up with a matrix with permuted columns, see the example (2.26).
} 
This form of $\mathcal{G}$ also immediately gives us a solution for the dependent variables:

$$
t_{i}^{(D)}=-\sum_{j=1}^{N_{I}} a_{i j} t_{i}^{(I)} .
$$

This expression can be substituted into (2.12), so that we obtain equations only for the independent variables, $f_{i}\left(t^{(I)}, t^{(D)}\left(t^{(I)}\right)\right)$.

However, these equations are still overdetermined, so in order to solve them, we first have to select the projector $P$. In principle, many choices are possible as long as the projector has the correct rank $N_{I}$. For example, Kishimoto and Takahashi $[39,40]$ used the Siegel gauge projector in their calculations in the a-gauge. However, there is one canonical choice for the projector.

The most natural choice for the projected equations is obtained by substituting the dependent variables into the action, $S(t) \rightarrow S\left(t^{(I)}, t^{(D)}\left(t^{(I)}\right)\right)$, and by taking derivatives of this restricted action with respect to the independent variables,

$$
\frac{\partial S\left(t^{(I)}, t^{(D)}\left(t^{(I)}\right)\right)}{\partial t_{i}^{(I)}}=0 .
$$

Using (2.11) and (2.17), we can derive an explicit formula for the canonical projector $P_{C}$. It is closely related to the transpose of the matrix $\mathcal{G}$ :

$$
P_{C}=\left(\begin{array}{cccccccc}
1 & 0 & \ldots & 0 & -a_{11} & -a_{21} & \ldots & -a_{N_{D} 1} \\
0 & 1 & \ldots & 0 & -a_{12} & -a_{22} & \ldots & -a_{N_{D} 2} \\
\vdots & \vdots & \ddots & \vdots & \vdots & \vdots & \ddots & \vdots \\
0 & 0 & \ldots & 1 & -a_{1 N_{I}} & -a_{2 N_{I}} & \ldots & -a_{N_{D} N_{I}}
\end{array}\right) .
$$

If one decides to use the canonical projector, which we do in this work, one can in principle avoid explicit use of the projector by working directly with the restricted action as in [36]. However, it is not possible to construct the full matrix representation of the cubic vertex $V_{i j k}$ at high levels due to large memory requirements, and we have to work with the factorized matrices $V_{i j k}^{\text {matter }}$ and $V_{i j k}^{\text {ghost }}$ only. The projector is not compatible with the factorized vertices, therefore we have to apply it directly in Newton's method.

When we work out Newton's method for the projected equations (2.18), we find that the crucial step changes to

$$
\sum_{j} M_{i j}^{(P)}\left(t^{(n)}\right) \Delta t_{j}^{(I)}=-f_{i}^{(P)}\left(t^{(n)}\right)
$$

where we define the projections of the Jacobian matrix and of the equations of motion in terms of the non-projected quantities as

$$
\begin{aligned}
M_{i j}^{(P)} & =\sum_{k, l} P_{i k} P_{C j l} M_{k l}, \\
f_{i}^{(P)} & =\sum_{j} P_{i j} f_{j} .
\end{aligned}
$$


One of the steps of Newton's method allows us to find the change of the independent variables, so the dependent variables can be then easily computed using (2.17). Notice that, if one decides to use a non-canonical projector, the Jacobian is multiplied by a different projector from each side.

As a matter of illustration, using a truncated level 4 string field, we would like to explain in some detail how the method above works. The string field up to level 4 , following the notation of Sen and Zwiebach [41], is given by

$$
\begin{aligned}
|\Psi\rangle= & t c_{1}|0\rangle+u c_{-1}|0\rangle+v L_{-2}^{m} c_{1}|0\rangle+w b_{-2} c_{0} c_{1}|0\rangle+A L_{-4}^{m} c_{1}|0\rangle+B L_{-2}^{m} L_{-2}^{m} c_{1}|0\rangle \\
& +C c_{-3}|0\rangle+D b_{-3} c_{-1} c_{1}|0\rangle+E b_{-2} c_{-2} c_{1}|0\rangle+F L_{-2}^{m} c_{-1}|0\rangle+w_{1} L_{-3}^{m} c_{0}|0\rangle \\
& +w_{2} b_{-2} c_{-1} c_{0}|0\rangle+w_{3} b_{-4} c_{0} c_{1}|0\rangle+w_{4} L_{-2}^{m} b_{-2} c_{0} c_{1}|0\rangle
\end{aligned}
$$

which means that the vector $t_{i}$ consists of the following coefficients:

$$
t_{i}=\left(t, u, v, w, A, B, C, D, E, F, w_{1}, w_{2}, w_{3}, w_{4}\right) .
$$

The Schnabl gauge condition $\mathcal{B}_{0}|\Psi\rangle=0$ at this level contains 5 independent equations

$$
\begin{aligned}
w+\frac{2}{3} E & =0, \\
w_{i} & =0, \quad i=1,2,3,4 .
\end{aligned}
$$

The matrix of gauge conditions $\mathcal{G}$ that follows from (2.25) reads

$$
\mathcal{G}=\left(\begin{array}{llllllllllllll}
0 & 0 & 0 & 1 & 0 & 0 & 0 & 0 & \frac{2}{3} & 0 & 0 & 0 & 0 & 0 \\
0 & 0 & 0 & 0 & 0 & 0 & 0 & 0 & 0 & 0 & 1 & 0 & 0 & 0 \\
0 & 0 & 0 & 0 & 0 & 0 & 0 & 0 & 0 & 0 & 0 & 1 & 0 & 0 \\
0 & 0 & 0 & 0 & 0 & 0 & 0 & 0 & 0 & 0 & 0 & 0 & 1 & 0 \\
0 & 0 & 0 & 0 & 0 & 0 & 0 & 0 & 0 & 0 & 0 & 0 & 0 & 1
\end{array}\right) .
$$

The matrix has reordered columns compared to (2.16) because we ordered the columns following (2.24). We can easily read off the dependent and independent variables,

$$
\begin{aligned}
t_{i}^{(I)} & =(t, u, v, A, B, C, D, E, F), \\
t_{i}^{(D)} & =\left(w, w_{1}, w_{2}, w_{3}, w_{4}\right) .
\end{aligned}
$$

Then we construct the canonical projector (2.19)

$$
P_{C}=\left(\begin{array}{cccccccccccccc}
1 & 0 & 0 & 0 & 0 & 0 & 0 & 0 & 0 & 0 & 0 & 0 & 0 & 0 \\
0 & 1 & 0 & 0 & 0 & 0 & 0 & 0 & 0 & 0 & 0 & 0 & 0 & 0 \\
0 & 0 & 1 & 0 & 0 & 0 & 0 & 0 & 0 & 0 & 0 & 0 & 0 & 0 \\
0 & 0 & 0 & 1 & 0 & 0 & 0 & 0 & 0 & 0 & 0 & 0 & 0 & 0 \\
0 & 0 & 0 & 0 & 0 & 1 & 0 & 0 & 0 & 0 & 0 & 0 & 0 & 0 \\
0 & 0 & 0 & 0 & 0 & 0 & 1 & 0 & 0 & 0 & 0 & 0 & 0 & 0 \\
0 & 0 & 0 & 0 & 0 & 0 & 0 & 1 & 0 & 0 & 0 & 0 & 0 & 0 \\
0 & 0 & 0 & 0 & -\frac{2}{3} & 0 & 0 & 0 & 1 & 0 & 0 & 0 & 0 & 0 \\
0 & 0 & 0 & 0 & 0 & 0 & 0 & 0 & 0 & 1 & 0 & 0 & 0 & 0
\end{array}\right) .
$$


At this level, the projector has only one nontrivial element $P_{85}=-\frac{2}{3}$. The percentage of nonzero elements is very low even at higher levels, so we can work with the nontrivial elements only and forget the rest of the matrix.

Unfortunately, we can not illustrate explicitly how the projector acts on Newton's method because it would take far too much space, but we will at least argue that it reproduces the correct equations. So let us consider the expression

$$
\sum_{j} P_{C i j} \frac{\partial S(t)}{\partial t_{j}}=0
$$

and compare it to (2.18). The action of the projector at level 4 is mostly trivial. The projector reproduces the original equations for variables $(t, u, v, A, B, C, D, F)$ and, after substituting (2.25), we obtain the same equations as in (2.18). The equations for variables $\left(w_{1}, w_{2}, w_{3}, w_{4}\right)$ are correctly projected out, so the equations for $E$ and $w$ are the only nontrivial check. The projector mixes them together as

$$
\frac{\partial S}{\partial E}-\frac{2}{3} \frac{\partial S}{\partial w}=0
$$

It follows from (2.18) that

$$
\begin{aligned}
0 & =\frac{\partial S(E, w(E), \ldots)}{\partial E} \\
& =\frac{\partial S}{\partial E}+\frac{\partial w(E)}{\partial E} \frac{\partial S}{\partial w} \\
& =\frac{\partial S}{\partial E}-\frac{2}{3} \frac{\partial S}{\partial w},
\end{aligned}
$$

from which we observe that the projector reproduces the correct equation.

For consistency, we have checked up to level 10 that this method provides the same solution for tachyon vacuum in the Schnabl gauge as the approach used in [36]. Furthermore, we improved the results from [36] to level 24.

\section{Coefficients of the tachyon condensate}

Using $(L, 3 L)$ level truncation computations, we have determined the tachyon condensate in the Schnabl gauge up to level $L=24$. For practical purposes, we cannot provide the complete list of all the coefficients of the tachyon condensate up to this level 24 (there are 54678 coefficients). Instead, we show in table 2 the tachyon coefficient together with some other low level coefficients. We would like to compare these coefficients with the analytic solution from [2]. To do so, we will predict the asymptotic values of these coefficients using the methods studied in appendix A.

For a matter of illustration, we will explain in some detail the analysis of the data for the tachyon coefficient $t$. For the rest of the coefficients, we will only provide the results.

Let us start with the extrapolation of the tachyon coefficient data by means of functions in $1 / L$ of the form (A.1)

$$
T_{M}^{\left(L_{\min }, L_{\max }\right)}(L)=a_{0}+\sum_{n=1}^{M} \frac{a_{n}}{L^{n}},
$$




\begin{tabular}{|c|lllll|}
\hline$L$ & $t$ & $u$ & $v$ & $w$ & $w_{1}$ \\
\hline 2 & 0.544204232 & 0.190190387 & 0.055963664 & 0 & 0 \\
4 & 0.548938521 & 0.242291544 & 0.069463998 & -0.022500108 & 0 \\
6 & 0.548315148 & 0.271907177 & 0.077794757 & -0.036964272 & -0.0001097937 \\
8 & 0.547321883 & 0.291164859 & 0.083458462 & -0.046949656 & -0.0001568187 \\
10 & 0.546508411 & 0.304819814 & 0.087592014 & -0.054296587 & -0.0001789026 \\
12 & 0.545894444 & 0.315095030 & 0.090767237 & -0.059969107 & -0.0001895219 \\
14 & 0.545435158 & 0.323166082 & 0.093300578 & -0.064511081 & -0.0001943355 \\
16 & 0.545089161 & 0.329713368 & 0.095381100 & -0.068251134 & -0.0001960127 \\
18 & 0.544825972 & 0.335158842 & 0.097128893 & -0.071399574 & -0.0001959212 \\
20 & 0.544624075 & 0.339778735 & 0.098624073 & -0.074097466 & -0.0001948079 \\
22 & 0.544468282 & 0.343761952 & 0.099922283 & -0.076443182 & -0.0001931002 \\
24 & 0.544347732 & 0.347242371 & 0.101063472 & -0.078507578 & -0.0001910522 \\
\hline$L \rightarrow \infty$ & 0.54572 & 0.409 & 0.122 & -0.117 & -0.0000910 \\
\hline$N \rightarrow \infty$ & 0.55340 & 0.431 & 0.130 & -0.132 & 0.0000033 \\
\hline Analytic & 0.55346558 & 0.45661043 & 0.13764616 & -0.14421001 & 0 \\
\hline
\end{tabular}

Table 2. Some coefficients of the numerical solution in the Schnabl gauge up to level 24. We show coefficients of the level 2 string field plus the coefficient $w_{1}$ from level 4 . These coefficients are denoted following (2.23). The last three rows show the results of: $L \rightarrow \infty$ extrapolations (using level $(4,24)$ data and order $N=10), N \rightarrow \infty$ extrapolations (using order $M=5$ ), and the expected analytical values respectively.

where we are using the notation introduced in appendix A. Assuming that we have data from level $L_{\min }$ to $L_{\max }$, the order $M$ can be chosen between 1 and $\left(L_{\max }-L_{\min }\right) / 2$. For reasons explained in appendix A, we will use mainly polynomials of maximal orders $N=\left(L_{\max }-L_{\min }\right) / 2$.

The antepenultimate row and the second column of table 2 shows the asymptotic value of the tachyon coefficient $T_{10}^{(4,24)}(\infty) \approx 0.5457$, which has been obtained using data from levels $(4,24)$ and maximal order $N=10$ fit. $^{2}$ We find a rough agreement between this asymptotic value and the analytical value $t_{\text {analytic }}=0.55346558$, however, the precision is not sufficiently good. Let us see if we can improve the precision. The second column in table 3 shows the asymptotic values $\lim _{L \rightarrow \infty} T_{N}^{(4,2 N+4)}(L)$ obtained by means of maximal order extrapolations with varying number of data points. We observe that the results change nontrivially as we add more points and the general trend is that they approach the analytical value with increasing $N$. Therefore is worth trying to make a second, $N \rightarrow \infty$, extrapolation following the procedure described in appendix A.

By extrapolating the ten data points given in table 3, we can get a result that is much closer to the expected analytical one. Let us see explicitly how this works. To extrapolate

\footnotetext{
${ }^{2}$ In this section, we have decided to remove level 2 data from the analysis because they lead to lesser stability of $N \rightarrow \infty$ extrapolations. The most likely reason is that the solution at this level is the same as in the Siegel gauge.
} 


\begin{tabular}{|c|lllcc|}
\hline$N$ & $t$ & $u$ & $v$ & $w$ & $w_{1}$ \\
\hline 1 & 0.547068 & 0.331138 & 0.094456 & -0.065893 & -0.000109794 \\
2 & 0.541616 & 0.366737 & 0.106443 & -0.087919 & -0.000297894 \\
3 & 0.541628 & 0.380829 & 0.111773 & -0.097807 & -0.000221255 \\
4 & 0.542646 & 0.388399 & 0.114684 & -0.103198 & -0.000165502 \\
5 & 0.543345 & 0.393854 & 0.116718 & -0.106952 & -0.000138074 \\
6 & 0.543882 & 0.398126 & 0.118282 & -0.109836 & -0.000122554 \\
7 & 0.544389 & 0.401572 & 0.119533 & -0.112142 & -0.000110590 \\
8 & 0.544876 & 0.404346 & 0.120537 & -0.113988 & -0.000102323 \\
9 & 0.545315 & 0.406788 & 0.121407 & -0.115594 & -0.000091993 \\
10 & 0.545722 & 0.408636 & 0.122077 & -0.116808 & -0.000090974 \\
\hline
\end{tabular}

Table 3. Asymptotic values of the tachyon coefficient and the other coefficients from table 2. The results were obtained using functions $T_{N}^{(4,2 N+4)}(L)$ (and the analogues for the other coefficients) with $N$ varying from 1 to 10 .

the data in table 3 , we employ the following function:

$$
t_{M}(N)=r_{0}+\sum_{n=1}^{M} \frac{r_{n}}{N^{n}},
$$

where the coefficients $r_{0}, r_{1}, \cdots, r_{M}$ can be determined using the least squares fitting technique. In principle, the value of the fit degree $M$ could be chosen from 1 and 9 . However, the values in table 3 do not lie on a smooth curve like the original data. Therefore, for high values of $M$, it turns out that the coefficients $r_{0}, r_{1}, \cdots, r_{M}$ have large values and the extrapolations are unstable.

In the second column of table 4, we show the results of these extrapolations for the tachyon coefficient $\lim _{N \rightarrow \infty} t_{M}(N)$ for $2 \leq M \leq 7$. The results are not too far from each other, which means that for lower $M$, this extrapolation procedure is relatively stable. By repeating the same analysis for other coefficients, we have reached a conclusion that $M=5$ is the best overall choice, so these results are shown in the penultimate row of table 2 denoted by $N \rightarrow \infty$. For the tachyon coefficient, the result of the $M=5$ extrapolation agrees very well with the analytical value. The precision is about $0.01 \%$, but, considering that the other values in table 4 are further away, such good precision is probably coincidental.

When it comes to other coefficients, we decided to show the coefficients from level 2 string field, $u, v$ and $w$, and the coefficient $w_{1}$ from level 4 , which is equal to zero for the analytic solution. We analyzed them in the same way as the tachyon coefficient, the results are shown in tables 2, 3 and 4 in columns three to six. We can conclude that these coefficients behave similarly as the tachyon coefficient. The level infinity extrapolations roughly agree with the analytic values, but the precision is low. Therefore we again performed the $N \rightarrow \infty$ extrapolations, whose results are much closed to the expected analytic values, although the precision is lower than in the case of the tachyon coefficient. 


\begin{tabular}{|c|llllr|}
\hline$M$ & $t$ & $u$ & $v$ & $w$ & \multicolumn{1}{c|}{$w_{1}$} \\
\hline 2 & 0.547650 & 0.419700 & 0.126421 & -0.124876 & 0.000020124 \\
3 & 0.549081 & 0.425743 & 0.128172 & -0.128001 & -0.000052372 \\
4 & 0.549252 & 0.432140 & 0.130259 & -0.131814 & -0.000073693 \\
5 & 0.553396 & 0.431237 & 0.130247 & -0.131695 & 0.000003298 \\
6 & 0.553639 & 0.428358 & 0.129141 & -0.129134 & -0.000121455 \\
7 & 0.548023 & 0.416816 & 0.125705 & -0.120888 & -0.000821313 \\
\hline Analytic & 0.553466 & 0.456610 & 0.137646 & -0.144210 & 0 \\
\hline
\end{tabular}

Table 4. The results of $N \rightarrow \infty$ extrapolations of the data from table 3 using $1 / N$ polynomials of order $M$.

Finally, we would like to comment more about high level behavior of the tachyon coefficient $t$. In the reference [36], using results up to level $L=10$, the authors predicted that the tachyon coefficient reaches a local minimum value at level $L \sim 26$, to then turn back to approach the expected analytical result as $L \rightarrow \infty$. Using the additional data up to level 24, we will revise this result and show that this local minimum probably exists, but at a higher level.

If we assume that the numerical solution converges to the analytic one, the existence of the minimum is given by the fact that the values of the tachyon coefficient $t$ (see table 2) are below the correct result and keep going down. We can estimate at what level this local minimum arises using the functions $T_{8}^{(4,20)}(L), T_{9}^{(4,22)}(L)$ and $T_{10}^{(4,24)}(L)$. In figure 1, we plot these functions together with the tachyon coefficient data. And for reference, we also plot the function $T_{11}^{(2,24)}(L)$ that uses the maximal amount of data points. We have computed the local minimum of $T_{8}^{(4,20)}(L), T_{9}^{(4,22)}(L)$ and $T_{10}^{(4,24)}(L)$ and obtained the corresponding values $L \sim 46, L \sim 43$ and $L \sim 42$ respectively.

Since the position of the minimum changes as we include data from more levels and the corresponding fits, when extrapolated to level infinity, do not reproduce the analytical value well enough, these estimates of the position of the minimum might not be entirely trustworthy. We notice that the estimates decrease as we add more data points, so we can conjecture that the minimum lies few levels below level $L \lesssim 42$. We cannot make a precise prediction with the available data, but, from the trend observed in the data up to level 24 (see figure 1), it is very unlikely that this minimum would happen at $L=26$ as claimed in [36].

\section{The vacuum energy and the gauge invariant overlap}

The values for the vacuum energy obtained by direct $(L, 3 L)$ level truncation computations in the Schnabl gauge up to level $L=24$, are given in table 5. For purposes of comparison, we have also written the data for the vacuum energy in the Siegel gauge up to level $L=30$ [37].

In the reference [36], the energy in the Schnabl gauge up to level $L=10$ was computed using direct $(L, 3 L)$ level truncation computations and, by extrapolating this level $L=10$ data, it was predicted that the vacuum energy reaches a local minimum at level $L \sim 12$, to 


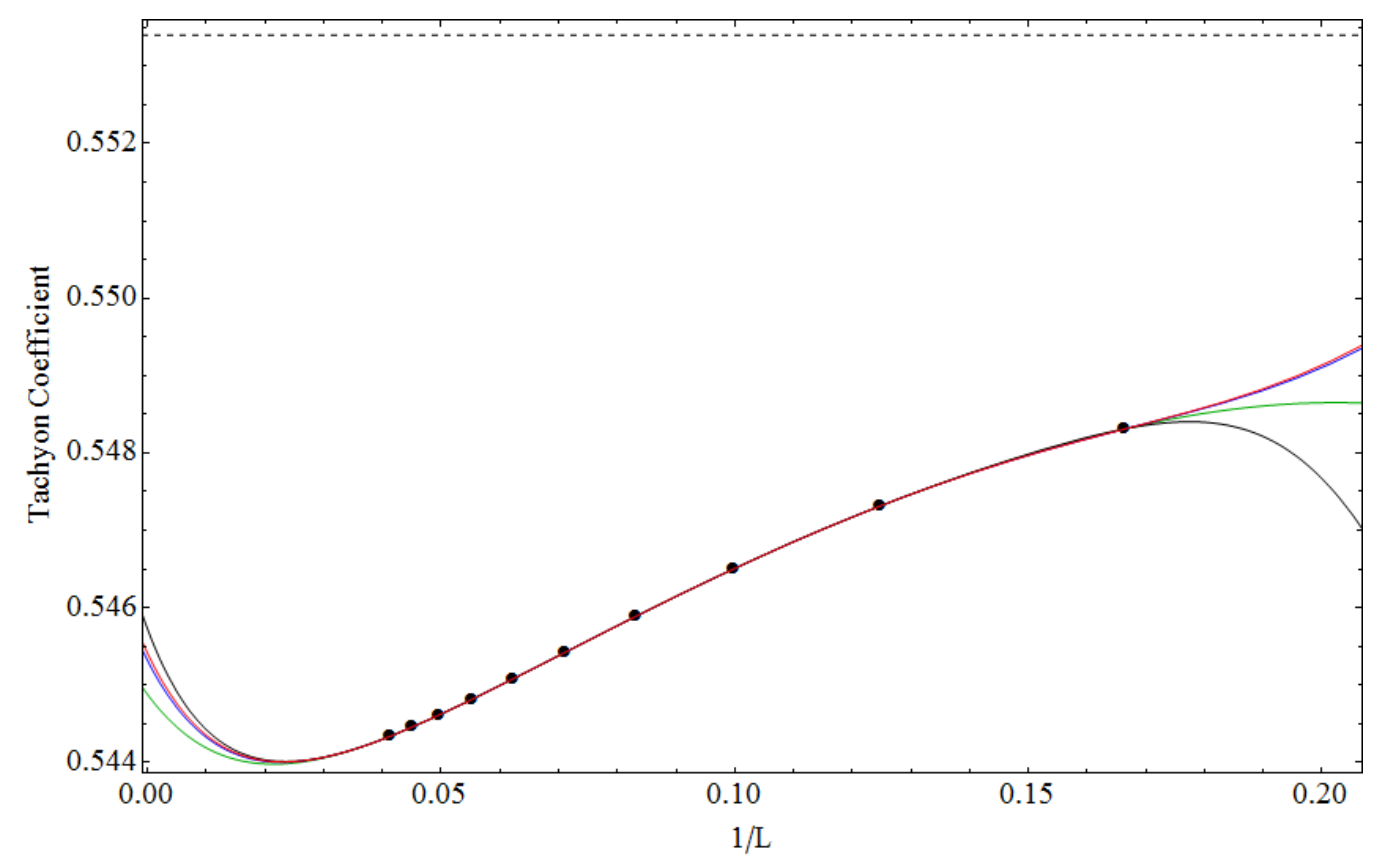

Figure 1. The tachyon coefficient data up to level $L=24$ in the Schnabl gauge as a function of $1 / L$. The green solid curve represents the function $T_{8}^{(4,20)}(L)$. The blue solid curve represents the function $T_{9}^{(4,22)}(L)$. The black solid curve represents the function $T_{10}^{(4,24)}(L)$. The red solid curve represents the function $T_{11}^{(2,24)}(L)$. The dashed line represents the analytical value 0.553465 .

\begin{tabular}{|c|c|c|}
\hline$L$ & $E^{\text {Sch }}$ & $E^{\text {Sie }}$ \\
\hline 2 & -0.959376599521 & -0.959376599521 \\
4 & -0.994651904750 & -0.987821756244 \\
6 & -1.003983765388 & -0.995177120537 \\
8 & -1.007110280219 & -0.997930183378 \\
10 & -1.008189759705 & -0.999182458475 \\
12 & -1.008466266815 & -0.999822263312 \\
14 & -1.008396790194 & -1.000173729946 \\
16 & -1.008173012946 & -1.000375451894 \\
18 & -1.007882751544 & -1.000493711466 \\
20 & -1.007568595810 & -1.000562954585 \\
22 & -1.007251843369 & -1.000602262320 \\
24 & -1.006943179985 & -1.000622749436 \\
26 & & -1.000631156455 \\
28 & & -1.000631706784 \\
30 & & -1.000627117967 \\
\hline$\infty$ & -0.99949 & -1.000009 \\
\hline
\end{tabular}

Table 5. $(L, 3 L)$ level-truncation results for the vacuum energy in the Schnabl gauge $E^{\text {Sch }}$ as well as in the Siegel gauge $E^{\text {Sie }}$. 


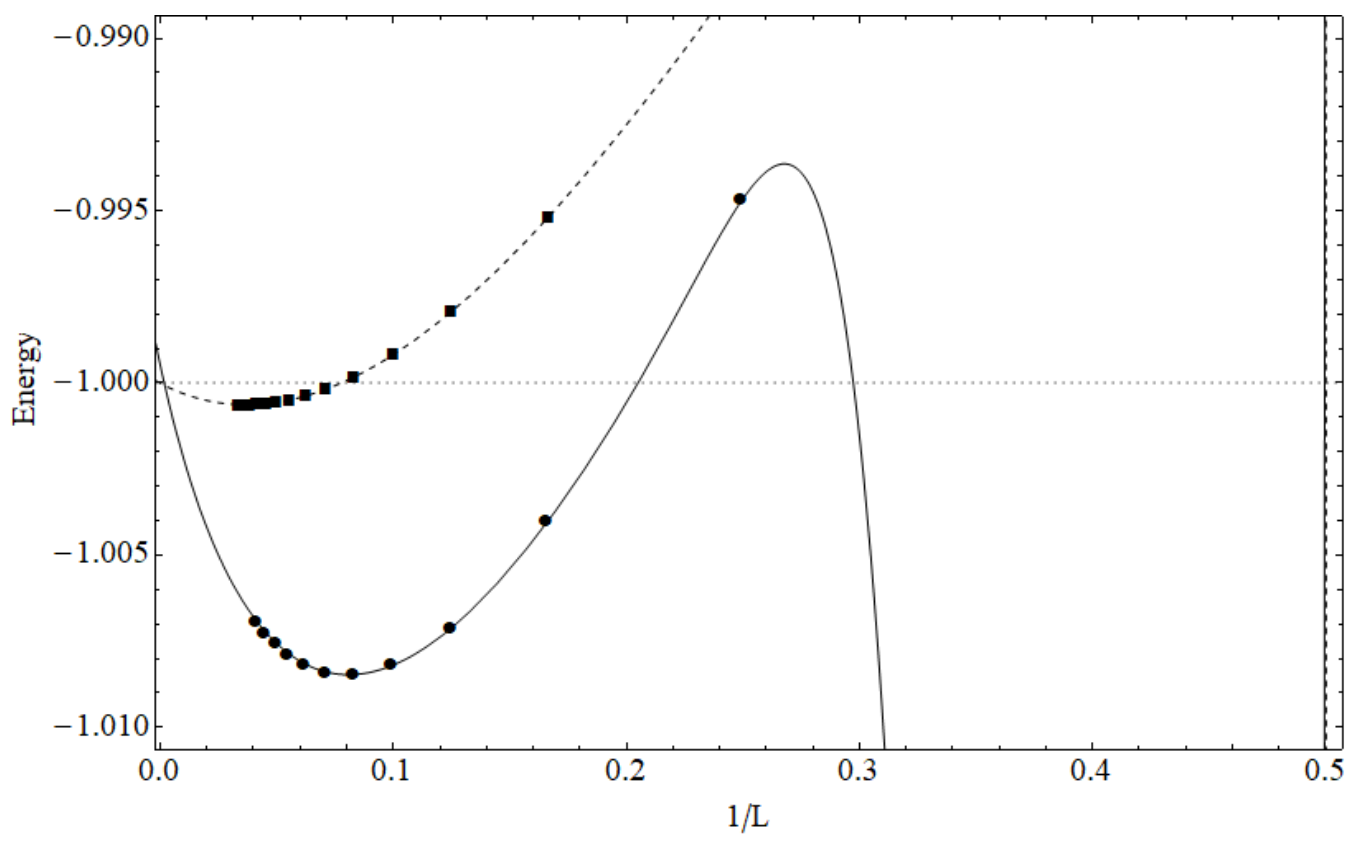

Figure 2. Data for the energy in the Schnabl gauge as well as in the Siegel gauge as a function of $1 / L$. The solid curve represents the function $E_{11}^{(2,24)}$ that interpolates the level $L=24$ data in the Schnabl gauge. The dashed curve represents the function $E_{14}^{(2,30)}$ that interpolates the level $L=30$ data in the Siegel gauge. The dotted line represents the analytical value -1 .

then turn back to approach its expected analytical value $-1,{ }^{3}$ asymptotically as $L \rightarrow \infty$. Note that the existence of this local minimum can be confirmed by looking directly at the data given in table 5 .

Since now we have data for the energy up to level $L=24$, it would be nice to see what the behavior of the energy at higher levels is. In order to extrapolate our data, we use the procedure introduced in appendix A. We consider polynomial functions of the form (A.1), where we choose the maximal order $N=\left(L_{\max }-L_{\min }\right) / 2$.

Employing the data given in table 5, we can construct functions $E_{N}^{(2,2 N+2)}(L)$ up to order $N=11$ in the Schnabl gauge, and up to order $N=14$ in the Siegel gauge. The asymptotic values $\lim _{L \rightarrow \infty} E_{N}^{(2,2 N+2)}(L)$ in both gauges are provided in table 6 . In figure 2, we show the plot of $E_{11}^{(2,24)}$ in the Schnabl gauge and of $E_{14}^{(2,30)}$ in the Siegel gauge. We observe that the precision of the fit in the Siegel gauge is much better. Even though the function $E_{11}^{(2,24)}$ in the Schnabl gauge fits the corresponding data well, we have noted that, at a level close to $L \sim 590$, the value of the extrapolated energy overshoots the expected result -1 and the asymptotic value when the level goes to infinity is close to -0.9995 . Other functions $E_{N}^{(2,2 N+2)}(L)$ with high $N$ behave similarly.

We note that the size of this overshooting is similar to the dispersion of the results, and therefore we can say that it lies within the error of the extrapolation procedure. Have

\footnotetext{
${ }^{3}$ The energy in this section is normalized according to the convention adopted in reference [36]. Note that, to match the convention adopted in this paper with the convention of [37], the value of the energy should be shifted by 1 .
} 


\begin{tabular}{|c|c|c|}
\hline$N$ & $E^{\text {Sch }}$ & $E^{\text {Sie }}$ \\
\hline 1 & -1.029927 & -1.0162669 \\
2 & -1.019008 & -1.0066983 \\
3 & -1.007440 & -1.0010884 \\
4 & -1.003143 & -1.0001414 \\
5 & -1.001520 & -1.0000732 \\
6 & -1.000607 & -1.0000552 \\
7 & -1.000045 & -1.0000371 \\
8 & -0.999722 & -1.0000256 \\
9 & -0.999568 & -1.0000178 \\
10 & -0.999476 & -1.0000137 \\
11 & -0.999486 & -1.0000131 \\
12 & & -1.0000096 \\
13 & & -1.0000094 \\
14 & & -1.0000091 \\
\hline
\end{tabular}

Table 6. Extrapolations of the energy using order $N$ polynomials and level $(2,2 N+2)$ data in the Schnabl and in the Siegel gauge.

a closer look at table 6. As in the previous section, there is a problem with stability of the extrapolations. We observe that the differences between these asymptotic values are much higher in the Schnabl gauge than in the Siegel gauge, and that these changes are not random, but they follow a certain trend. Similar behavior can be observed if we make other variations in the extrapolation procedure, for example, we can keep $L_{\max }=24$ and vary $L_{\min }$ or we can use less than maximal order extrapolations.

The simplest conclusion is that, based on the differences in table 6 , the asymptotic value of the energy in the Schnabl gauge has an error of order $10^{-4}$ and therefore the energy agrees with the analytical value within this error.

We have tried to make a second, $N \rightarrow \infty$ extrapolation of the values in table 6 similarly to section 3 , but the results do not lead to a better precision. Diverse extrapolations in $1 / N$ agree on a maximum around $N=10$, which means that these functions start to approach -1 again above this value, but the asymptotic values of these functions have too large dispersion to be trustworthy.

Another quantity analyzed is the so-called gauge invariant overlap or Ellwood's invariant. For a given solution $\Psi$ of the string field equation of motion, let us define this gauge invariant quantity $E_{0}$ as $^{4}$

$$
E_{0}=1+\langle\mathcal{V} \mid \Psi\rangle=1+\langle I|\mathcal{V}(i)| \Psi\rangle
$$

\footnotetext{
${ }^{4}$ We are using the same convention for $E_{0}$ as given in reference [37], which is shifted by -1 when compared with the convention of references $[43,44]$. The invariant can be easily evaluated using the conservation laws in [45].
} 


\begin{tabular}{|c|l|}
\hline$L$ & $E_{0}$ \\
\hline 2 & 0.110138182891 \\
4 & 0.068341344418 \\
6 & 0.047847933137 \\
8 & 0.034175803769 \\
10 & 0.027266149826 \\
12 & 0.020987152232 \\
14 & 0.017848318168 \\
16 & 0.014361780518 \\
18 & 0.012688927165 \\
20 & 0.010510916529 \\
22 & 0.009525064795 \\
24 & 0.008051965768 \\
\hline$\infty$ & -0.0015 \\
\hline
\end{tabular}

Table 7. $(L, 3 L)$ level-truncation results for the gauge invariant overlap $E_{0}$ in the Schnabl gauge.

where $|I\rangle$ is the identity string field and $\mathcal{V}(i)$ is an on-shell closed string vertex operator $\mathcal{V}=c \tilde{c} V^{\mathrm{m}}$, which is inserted at the midpoint of the string field $\Psi$. Using the $(L, 3 L)$ level truncated numerical solution in the Schnabl gauge, we computed $E_{0}$ up to level $L=24$. The results are shown in table 7 . Note that $E_{0}$ appears to approach the expected analytical value $E_{0}=0$.

Let us predict the value for this gauge invariant quantity $E_{0}$ when $L \rightarrow \infty$. To do this, we need to extrapolate the corresponding data given in table 7 . If we use the $1 / L$ polynomial interpolation given by equation (A.1) and the data of table 7, we can obtain an order $N=11$ extrapolation. However when we compute the asymptotic value $L \rightarrow \infty$ of this order $N=11$ function, we get 3.3629, which is clearly far away from the expected value $E_{0}=0$. So, we observe that for the gauge invariant overlap, we have to extrapolate levels $4 k+2$ and $4 k$ separately. ${ }^{5}$ Namely, we divide the twelve data points of table 7 into two sets of six data points, the ones at levels $L=(2,6,10,14,18,22)$ and $L=(4,8,12,16,20,24)$ and for each set of data points, we use a $1 / L$ polynomial function of order $N=5$. For instance, using the data of levels $L=4 k+2$, where $k=0,1, \cdots, 5$, we obtain the following result

$$
f_{5}(L)=-0.00172+\frac{0.159869}{L}+\frac{2.64626}{L^{2}}-\frac{18.1245}{L^{3}}+\frac{51.6933}{L^{4}}-\frac{51.0373}{L^{5}} .
$$

Note that when $L \rightarrow \infty$, the above expression approaches -0.00172 , which is close to the expected analytical value of the gauge invariant overlap, $E_{0}=0$. Now, if we use the data of levels $L=4 k$, where $k=1, \cdots, 6$, we obtain

$$
g_{5}(L)=-0.00129+\frac{0.129984}{L}+\frac{3.13195}{L^{2}}-\frac{24.3127}{L^{3}}+\frac{88.936}{L^{4}}-\frac{129.155}{L^{5}} .
$$

\footnotetext{
${ }^{5}$ See [38] for more detailed discussion of extrapolations of Ellwood invariants.
} 


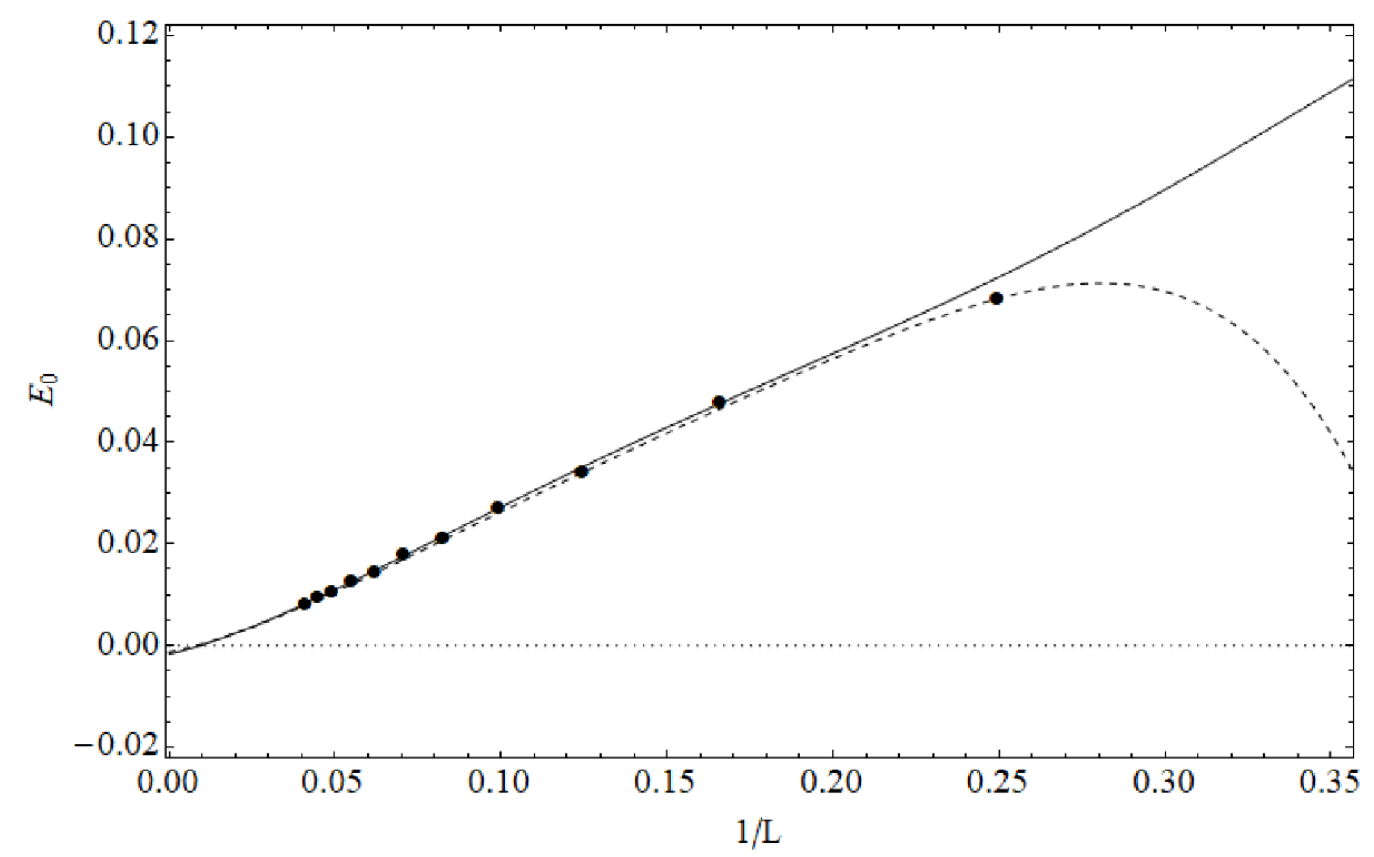

Figure 3. Data for the gauge invariant quantity $E_{0}$ up to level $L=24$ in the Schnabl gauge as a function of $1 / L$. The solid curve represents the function $f_{5}(L)$, while the dashed curve represents the function $g_{5}(L)$. The dotted line represents the expected analytical value $E_{0}=0$.

So in this case, the asymptotic value turns out to be -0.00129 , which is also close to the expected value of $E_{0}=0$. By taking the average of these two asymptotic values, we get -0.0015 , which means that this extrapolation technique gives us the invariant $E_{0}$ with a relative precision of $0.15 \%$.

For illustrative purposes, we have plotted in figure 3 the data of the gauge invariant quantity given in table 7 together with the functions $f_{5}(L)$ and $g_{5}(L)$.

\section{Checking some symmetries}

In reference [2], the author showed that the analytical solution in the Schnabl gauge satisfies $K_{1}^{\text {matter }} \Psi=0$ and $\left[K_{1}^{\text {matter }}, Q\right] \Psi=0$, where $K_{1}^{\text {matter }}=L_{1}^{\text {matter }}+L_{-1}^{\text {matter }}$. A consequence of this symmetry is as follows: consider the expansion of the analytical solution $\Psi$ in the Virasoro basis of $L_{0}$ eigenstates, for instance, this expansion up to level $L=4$ is given in equation (2.23), where the exact coefficients are shown in table 8. Employing the symmetries $K_{1}^{\text {matter }} \Psi=0$ and $\left[K_{1}^{\text {matter }}, Q\right] \Psi=0$, it is possible to show that the exact coefficients satisfy the following relations

$$
\begin{aligned}
w_{1} & =0, \\
d_{1} \equiv 5 A+3 B+v & =0, \\
d_{2} \equiv 20 A+12 B+4 D-4 F-8 w_{1} & =0, \\
d_{3} \equiv 15 A+9 B+v+w-10 w_{1}+5 w_{3}+3 w_{4} & =0 .
\end{aligned}
$$




\begin{tabular}{|llll|}
\hline$t=0.55346558$ & $A=-0.03027758$ & $E=0.17942652$ & $w_{1}=0$ \\
$u=0.45661043$ & $B=0.00458058$ & $F=0.02274827$ & $w_{2}=0.02094354$ \\
$v=0.13764616$ & $C=-0.16494614$ & & $w_{3}=0.08898226$ \\
$w=-0.14421001$ & $D=0.16039444$ & & $w_{4}=-0.00846965$ \\
\hline
\end{tabular}

Table 8. Coefficients of Schnabl analytical solution up to level 4.

\begin{tabular}{|c|lrcc|}
\hline$L$ & $d_{1}$ & \multicolumn{1}{c}{$d_{2}$} & \multicolumn{1}{c}{$d_{3}$} & \multicolumn{1}{c|}{$d_{4}$} \\
\hline 4 & 0.0418100836 & -0.0051417488 & -0.0359978557 & -0.0313612657 \\
6 & 0.0389000577 & 0.0004040458 & -0.0326194412 & -0.0311201802 \\
8 & 0.0368605116 & 0.0028520778 & -0.0305285804 & -0.0300137406 \\
10 & 0.0352699472 & 0.0041306703 & -0.0290140111 & -0.0286764813 \\
12 & 0.0339700353 & 0.0048583975 & -0.0278298640 & -0.0273399210 \\
14 & 0.0328764762 & 0.0052915301 & -0.0268613392 & -0.0260829188 \\
16 & 0.0319371821 & 0.0055535049 & -0.0260450246 & -0.0249272454 \\
18 & 0.0311173345 & 0.0057100850 & -0.0253419397 & -0.0238732195 \\
20 & 0.0303924516 & 0.0057989507 & -0.0247263128 & -0.0229134689 \\
22 & 0.0297446764 & 0.0058429156 & -0.0241801873 & -0.0220384197 \\
24 & 0.0291606055 & 0.0058564022 & -0.0236905443 & -0.0212384646 \\
\hline$L \rightarrow \infty$ & 0.0158 & 0.00256 & -0.0127 & -0.0031 \\
\hline$N \rightarrow \infty$ & 0.0074 & -0.00082 & -0.0059 & 0.0059 \\
\hline Analytic & 0 & 0 & 0 & 0 \\
\hline
\end{tabular}

Table 9. Results for the identities (5.2)-(5.5) obtained from the numerical solution in the Schnabl gauge up to level 24.

Using the values of the coefficients given in table 8 , we can explicitly verify that these coefficients satisfy the above identities. There is one more independent identity

$$
d_{4} \equiv 2 A+4 D-3 E+2 F-3 w_{2}+3 w_{4}=0 .
$$

In reference [2], the author failed in finding a simple origin for this identity, it might be just an accidental symmetry.

We would like to verify whether the numerical solution satisfies the identities (5.1)(5.5). Actually, the identity $w_{1}=0$ appears to be satisfied by the numerical solution when $L \rightarrow \infty$, see the last column of table 2 .

Using numerical results for the values of the coefficients that have been obtained by means of $(L, 3 L)$ level truncation computations up to level $L=24$ for the remaining identities (5.2)-(5.5), we found the results shown in table 9 .

Since these identities are linear combinations of string field coefficients, they can be extrapolated using the methods of appendix A. The line denoted by $L \rightarrow \infty$ shows results obtained by means of functions of the form (A.1) (in the case of the identities (5.2)-(5.5), we denote these functions as $\left.d_{i, 10}^{(4,24)}(L), i=1,2,3,4\right)$. The values shown in the line with 


\begin{tabular}{|c|lclll|}
\hline$L$ & $f_{w}$ & $f_{w_{1}}$ & $f_{w_{2}}$ & $f_{w_{3}}$ & $f_{w_{4}}$ \\
\hline 2 & 0.03332993 & -0.0748098 & 0.04390940 & 0.14787107 & 0.04589104 \\
4 & 0.01648496 & -0.1755207 & 0.00810403 & -0.03525052 & -0.02910861 \\
6 & 0.01026732 & -0.1129462 & 0.00682673 & -0.02057664 & -0.01258739 \\
8 & 0.00719823 & -0.0830693 & 0.00595911 & -0.01393171 & -0.00577056 \\
10 & 0.00541301 & -0.0653714 & 0.00528328 & -0.01023858 & -0.00240404 \\
12 & 0.00425955 & -0.0536435 & 0.00474400 & -0.00791965 & -0.00053883 \\
14 & 0.00345867 & -0.0453054 & 0.00430652 & -0.00634174 & 0.00057987 \\
16 & 0.00287305 & -0.0390806 & 0.00394589 & -0.00520561 & 0.00128963 \\
18 & 0.00242792 & -0.0342623 & 0.00364403 & -0.00435276 & 0.00175833 \\
20 & 0.00207934 & -0.0304272 & 0.00338784 & -0.00369181 & 0.00207654 \\
22 & 0.00179983 & -0.0273058 & 0.00316769 & -0.00316657 & 0.00229645 \\
24 & 0.00157136 & -0.0247185 & 0.00297641 & -0.00274064 & 0.00244972 \\
\hline$\infty$ & -0.00026 & 0.0012 & 0.00009 & 0.00045 & 0.00081 \\
\hline
\end{tabular}

Table 10. Violation of the equations of motion up to level 4.

$N \rightarrow \infty$ were obtained by fits of the form given in equation (A.2) with degree $M=5$. With the exception of the last identity $d_{4}$, the $N \rightarrow \infty$ extrapolations improve the results of the usual $L \rightarrow \infty$ extrapolations.

From the results given in table 9, we can conclude that the identities (5.2)-(5.5) are satisfied.

\section{Out-of-gauge equations of motion}

In the level truncation approach to SFT, it is possible to solve only a subset of the full equations of motion (2.5). However, in order to verify that the tachyon vacuum is a physical solution, we should check whether it violates the remaining equations (2.6). In the ideal case, the violation of these equations approaches zero as the level goes to infinity.

The evaluation of these out-of-gauge equations in the Schnabl gauge is much simpler than in the Siegel gauge because we have access to the full set of cubic vertices. Therefore we decided to check the equations that come from derivations of the action with respect to the dependable variables (2.28). The results are summarized in table 10, including extrapolations to infinite level.

We extrapolate the data using functions of the form (A.1), where we choose $L_{\min }=4$ and the maximal possible order $N=10$. We omit the data points at level 2 because they do not follow the trend given by the remaining data points. There are probably two reasons for that. The solution at level 2 is the same as in the Siegel gauge and the equations for level 4 fields cannot be satisfied well while we are still at level 2. We have also tried to do $N \rightarrow \infty$ extrapolations like in section 3 , but when we computed the analogue of table 3 , we have found that these results are not orderly enough to allow meaningful extrapolations in $1 / N$. 
The first equation $f_{w}$ is a direct analogue of $\Delta_{S}$ in [37]. We can see that it quickly decreases with the level, although somewhat slower than in case of the Siegel gauge solution. The other equations are also satisfied well and most of them monotonously approach zero (excluding the exceptional level 2). The extrapolations improve the values at level 24 by another order, so it is most likely that these equations are asymptotically satisfied. The only exception to this trend is the equation $f_{w_{4}}$, which overshoots zero. However, its extrapolation predicts that it has a maximum around level 37 to then turn back and go to zero as well.

\section{Summary and discussion}

In this work, we developed a technique that allows us to compute high level solutions in nontrivial gauges. We applied it on the tachyon vacuum solution in the Schnabl gauge and improved the results from [36] to level 24.

The overall behavior of energy of the Schnabl gauge solution is similar to the Siegel gauge. In particular, it overshoots the correct value, goes through a local minimum at level 12 and then it turns back towards -1 . By extrapolating the energy, we found that the solution satisfies the Sen's first conjecture with a precision of about $0.05 \%$. This is not a bad result, but it is worse than in the Siegel gauge. The gauge invariant $E_{0}$ converges towards the correct value monotonically and its infinite level extrapolation reproduces the analytic value with a precision of about $0.15 \%$.

An intriguing question is whether the numerical solution in fact converges to the Schnabl analytical solution. In this work, we reach a conceivable conclusion that it does, but additional effort will be needed to prove it with absolute certainty. At finite levels, there is an unexpectedly big difference between the two solutions. By employing various extrapolation techniques, we have found that the solution moves in the right direction and we can get much closer to the analytical solution, but the precision is still significantly lower than in the case of the gauge invariant observables.

In addition to straightforward comparisons of coefficients, we tested whether the numerical solution satisfies some identities valid for the analytical solution and the equations we projected out during the implementation of the Schnabl gauge. Both of these consistency checks are asymptotically satisfied quite well, which supports the claim that the numerical solution converges to the analytical one.

In general, extrapolations of the tachyon vacuum solution in the Schnabl gauge are less reliable than in the Siegel gauge. Most importantly, there is a partial instability because asymptotic values change significantly as we add new levels. This suggests that the Schnabl gauge solution has an unusual asymptotic behavior. It is possible that when the solution is expanded in $1 / L$ around infinity, the coefficients have slow falloff and the level 24 approximation is still not good enough. Another possibility is that the numerical solution is a non-analytic function around level infinity, and therefore one has to use a different asymptotic expansion.

In order to understand the origin of these problems, we have made a number of low level experiments with linear $b$-gauges. For instance, consider a simple gauge $\left(b_{0}+\alpha b_{2}\right) \Psi=0$. 
In gauges which are close to the Siegel gauge $(\alpha \rightarrow 0)$, tachyon vacuum solutions behave well, while when we go further away from the Siegel gauge, there are similar problems with convergence as in the Schnabl gauge. The Schnabl gauge solution is not special in this respect. Our best guess is that the problems with convergence are caused by the fact that the gauge condition couples states at different levels. If the coupling is too strong, the level truncation scheme becomes less reliable. We leave a detailed analysis of tachyon vacuum solution in these gauges as a future research project.

As another numerical experiment, we tried to find a tachyon vacuum solution using a different approach inspired by analytical $K B c$ solutions. Consider a generic $K B c$ string field

$$
\Psi=F_{1}(K) c F_{2}(K)+G_{1}(K) c G_{2}(K) B c G_{3}(K) .
$$

By level expansion of such string field, we find states of the form

$$
L_{-2 k_{1}}^{\mathrm{tot}} \ldots L_{-2 k_{n}}^{\mathrm{tot}} c_{-l}|0\rangle, \quad k_{i}>0, l \geq-1
$$

and

$$
L_{-2 k_{1}}^{\mathrm{tot}} \ldots L_{-2 k_{n}}^{\mathrm{tot}} b_{-2 m} c_{-l_{1}} c_{-l_{2}}|0\rangle, \quad k_{i}>0, l_{1,2} \geq-1, m>0
$$

We call the state space spanned by these states a restricted space. It can be described by linear constraints similar to (2.2) and therefore it is possible to search for SFT solutions in the restricted space using the same techniques as for the gauge fixing. The restricted space is not closed under string field theory operations (meaning that if $\Psi_{1}, \Psi_{2}$ belong to the restricted space, $Q^{-1}\left(\Psi_{1} * \Psi_{2}\right)$ does not), but one can hope that the projected out equations will be satisfied in the infinite level limit.

Unfortunately, our attempts to find solutions in this setup have not been successful. First, we tried not to impose any gauge. However, the restricted space conditions do not fix enough coefficients at low levels, we therefore encountered the same difficulties as in [37]. There are multiple solutions corresponding to the tachyon vacuum and when we try to improve them to higher levels, Newton's method fails.

Therefore, it appears necessary to impose some gauge condition in addition to the restricted space conditions. We tested both the Siegel and Schnabl gauge conditions, and both cases lead to similar results. There is a unique tachyon vacuum solution at low levels, but at higher levels, problems with the numerical stability start to appear. Actually, Newton's method fails to converge at level 20. Furthermore, the value of the energy associated to the numerical solution does not appear to converge to -1 and some of the projected out equations are not satisfied.

These results lead us to the conclusion that the $K B c$ algebra cannot be consistently truncated to finite levels. The restricted space conditions remove too many degrees of freedom at high levels (more than the gauge fixing), therefore the remaining fields cannot solve the SFT equation. Therefore the full analytical expressions are needed to work with the $K B c$ algebra. 


\section{Acknowledgments}

We would like to thank Ted Erler and Carlo Maccaferri for the useful discussions. This research was supported partly by the Czech Science Foundation (GAČR) grant 17-22899S and by the European Regional Development Fund and the Czech Ministry of Education, Youth and Sports (MŠMT), project No. CZ.02.1.01/0.0/0.0/15_003/0000437. Computational resources were provided by the CESNET LM2015042 and the CERIT Scientific Cloud LM2015085, provided under the programme "Projects of Large Research, Development, and Innovations Infrastructures.

\section{A Extrapolations to infinite level}

In this appendix, we provide general rules for extrapolations applied to the data of various quantities.

Suppose we have a set of data points for some quantity from level $L_{\min }$ to $L_{\max }{ }^{6} \mathrm{In}$ order to perform extrapolations, we will be required to fit this set of data points using functions of the form $[38,42]^{7}$

$$
q_{M}^{\left(L_{\min }, L_{\max }\right)}(L)=a_{0}+\sum_{n=1}^{M} \frac{a_{n}}{L^{n}},
$$

where the order of the fit $M$ can vary from $M_{\min }=1$ to its maximal value $M_{\max }=$ $\left(L_{\max }-L_{\min }\right) / 2$. We denote this maximal value as $M_{\max } \equiv N$. Note that the value of $N$ is equal to the number of used data points minus 1 . The coefficients $a_{0}, a_{1}, \cdots, a_{M}$ are determined by minimizing the sum of the squares of the residuals of the data points from the function (A.1). Namely, we use the least squares fitting technique. In practice, we use the function NonlinearModelFit in Mathematica.

In the case of the Siegel gauge, we usually use all available data points and maximal order fits to perform extrapolations. This procedure works very well, see [35] and also [38]. Let us analyze whether this approach also works in the Schnabl gauge. A reliable extrapolation procedure should be applicable to multiple quantities and it should satisfy the following properties:

1. It should approximate the data well.

2. It should be stable, namely its result should not change much if we add/remove a data point or change the order of the fit.

3. It should reproduce the analytical expected values.

\footnotetext{
${ }^{6}$ It is assumed that we have data points at even levels only. If we have data at odd levels (or possibly at fractional levels), the data points must be divided into groups, where the members of each group differ by two levels, and each group must be extrapolated separately, see [38] for more details. In the case of Ellwood invariants, the interval is four levels, so there are always at least two groups.

${ }^{7}$ It is also possible to try to use polynomials in $\frac{1}{L+1}$ as in [35], or even more generic functions using $\frac{1}{L+a}$ instead of $\frac{1}{L}$. However the results of the extrapolation procedure have only a small dependence on $a$, so we set $a=0$ for simplicity.
} 


\begin{tabular}{|c|c|c|c|c|c|c|c|}
\hline$L_{\min }$ & $L_{\max }$ & $M$ & $|\Delta|$ & $L_{\min }$ & $L_{\max }$ & $M$ & $|\Delta|$ \\
\hline \multicolumn{4}{|c|}{ Energy } & \multicolumn{4}{|c|}{$t$} \\
\hline 4 & 22 & 9 & $4.27 \times 10^{-10}$ & 2 & 22 & 10 & $2.15 \times 10^{-8}$ \\
\hline 6 & 22 & 8 & $8.38 \times 10^{-10}$ & 4 & 22 & 9 & $2.38 \times 10^{-8}$ \\
\hline 2 & 22 & 10 & $9.17 \times 10^{-10}$ & 6 & 22 & 8 & $2.94 \times 10^{-8}$ \\
\hline \multicolumn{4}{|c|}{$v$} & \multicolumn{4}{|c|}{$u$} \\
\hline 2 & 22 & 10 & $3.47 \times 10^{-8}$ & 2 & 22 & 10 & $9.55 \times 10^{-8}$ \\
\hline 4 & 22 & 9 & $3.92 \times 10^{-8}$ & 4 & 22 & 9 & $1.08 \times 10^{-7}$ \\
\hline 6 & 22 & 8 & $5.04 \times 10^{-8}$ & 6 & 22 & 8 & $1.39 \times 10^{-7}$ \\
\hline \multicolumn{4}{|c|}{$f_{w}$} & \multicolumn{4}{|c|}{$f_{w_{1}}$} \\
\hline 2 & 22 & 10 & $3.79 \times 10^{-9}$ & 2 & 22 & 10 & $1.68 \times 10^{-8}$ \\
\hline 4 & 22 & 9 & $4.20 \times 10^{-9}$ & 4 & 22 & 9 & $1.79 \times 10^{-8}$ \\
\hline 6 & 22 & 8 & $5.21 \times 10^{-9}$ & 6 & 22 & 8 & $2.08 \times 10^{-8}$ \\
\hline
\end{tabular}

Table 11. Predictions for level 24 data for various quantities in the Schnabl gauge. We consider all possible data sets from level $L_{\min }$ to $L_{\max }$, which go from 2 to 22 , and fits with all possible orders $M$. We show the best three results for all quantities, which suggest that it is best to use maximal order fits and as much data points as possible.

To test the first point, for a set of data corresponding to some quantity, we can check whether we can predict level 24 data using lower level data. We consider all possible data sets from level $L_{\min }$ to $L_{\max }$, which go from 2 to 22 , and fits with all possible orders $M$.

To determine which of the fits (A.1) provides the best prediction for the value of the quantity at level $L=24$, we evaluate $|\Delta| \equiv\left|q_{M}^{\left(L_{\min }, L_{\max }\right)}(24)-q(24)\right|$, which represents the absolute value of the residuals of the predicted value of the quantity from its actual value $q(24)$ that has been obtained by direct level truncation computations.

In table 11, we show what are the best three predictions of level 24 data for several different quantities, which include the energy, some string field coefficients and some outof-gauge equations. We can see that all the best fits use the maximal possible order and all or almost all data points. Therefore the procedure used in the Siegel gauge should work in the Schnabl gauge too. Note that these fits are interpolation functions that pass through the data points. This result suggests that the level truncation data lie on some smooth curve and they do not contain random fluctuations like experimental data (for instance, due to noise effects).

We observe that the coefficients $a_{n}$ grow quickly with $n$, both in the Siegel and Schnabl gauge. For $M=11$, the last coefficient is typically of order $10^{6}$. However this does not appear to indicate unreliability of extrapolations, but it is a feature required by the form of the function (A.1). The function is typically evaluated at high levels, where powers of $\frac{1}{L}$ are very small. For example the term $\frac{a_{11}}{L^{11}}$ at level 24 equals $6.6 \times 10^{-16} a_{11}$. Therefore the coefficient $a_{11}$ must be quite high to make the term even noticeable. 


\begin{tabular}{|cccc|cccc|}
\hline$L_{\min }$ & $L_{\max }$ & $M$ & $\infty$ & $L_{\min }$ & $L_{\max }$ & \multicolumn{5}{c|}{$f_{w_{1}}$} \\
\hline \multicolumn{3}{|c|}{$f_{w}$} & & \multicolumn{5}{c|}{} \\
\hline 4 & 24 & 10 & -0.00026 & 4 & 8 & 2 & 0.00091 \\
6 & 24 & 9 & -0.00027 & 2 & 12 & 5 & 0.00098 \\
8 & 24 & 8 & -0.00029 & 4 & 24 & 10 & 0.00119 \\
\hline \multicolumn{3}{|c|}{$f_{w_{2}}$} & \multicolumn{5}{c}{$f_{w_{3}}$} \\
\hline 4 & 24 & 10 & 0.000091 & 2 & 14 & 5 & 0.00002 \\
6 & 24 & 9 & 0.000094 & 2 & 16 & 5 & 0.00031 \\
8 & 24 & 8 & 0.000101 & 2 & 12 & 5 & -0.00044 \\
\hline
\end{tabular}

Table 12. The best three extrapolations for some out-of-gauge equations in the Schnabl gauge. We considered all possible data sets from level $L_{\min }$ to $L_{\max }$, which go from 2 to 24 , and fits of all possible orders $M$. Overall, the best choice of extrapolations of these equations is to use the data from levels 4 to 24 and the maximal order $N=10$. There are sometimes better agreements (for example for $f_{w_{3}}$, where the suggested fit is only the 4 th best), but these are clearly coincidental.

Regarding the stability of extrapolations, we observe that the results are worse than in the Siegel gauge and there is only a partial stability. For instance, see table 6, where we show asymptotic values of the energy that were computed using extrapolations of the form $E_{N}^{(2,2 N+2)}(L)$. Although the results do not change much when we change $N$ by one unit, the differences are bigger than in Siegel gauge. A similar behavior can be seen in the case of string field coefficients in table 3 .

We have not found any fits for the energy, as well as for the string field coefficients, that are free of this stability issue. This probably suggests that the numerical solution in the Schnabl gauge has some unusual asymptotic behavior.

In the case of other quantities, like the gauge invariant overlap and the out-of-gauge equations, we observed that their extrapolated values are more stable than those observed in the case of the energy. For the out-of-gauge equations, the best results are generally obtained by maximal order fits using data from level 4 , see table 12 .

Finally, regarding the third point, it turns out that the energy and, even more significantly, string field coefficients have lesser precision than we would like and there are essential differences between the extrapolated and the analytical expected values. Nevertheless, we observe that the extrapolations clearly move in some direction as we increase the order $N$. Therefore we can try to make a second, $N \rightarrow \infty$, extrapolation which potentially allows us to improve the results. So let us establish the procedure to determine this $N \rightarrow \infty$ extrapolation.

Suppose we have data points from level $L_{\min }$ to $2 N+L_{\min }$. As a first step, we construct functions of the form $q_{N}^{\left(L_{\min }, 2 N+L_{\min }\right)}(L)$ and evaluate their asymptotic values, namely we compute $q_{N} \equiv \lim _{L \rightarrow \infty} q_{N}^{\left(L_{\min }, 2 N+L_{\min }\right)}(L)$. 
As a second step, by means of polynomials in $1 / N$ of order $M$

$$
r_{0}+\sum_{n=1}^{M} \frac{r_{n}}{N^{n}},
$$

we fit the set of data points $\left\{q_{1}, q_{2}, \cdots, q_{N}\right\}$, where the coefficients $r_{0}, r_{1}, \cdots, r_{M}$ are again determined using the least squares fitting technique. Finally, we compute the asymptotic value of (A.2) when $N \rightarrow \infty$. In this extrapolation procedure, it turns out that it is necessary to use less than maximal order fits, because fits with high $M$ are unstable.

This second $N \rightarrow \infty$ extrapolation procedure works quite well for the string field coefficients from section 3 , for which we find that the best results are usually given by fits with $M=5$. While in the case of the energy and out-of-gauge equations, the differences between fits of different order $M$ are of similar size as the precision of original $L \rightarrow \infty$ extrapolations.

\section{B Search for other solutions}

Following [37], we also attempted to search for other Schnabl gauge solutions different from the tachyon vacuum. We used the homotopy continuation method ${ }^{8}$ adapted to Schnabl gauge to find all solutions of the equations of motion at low levels, which serve as seeds for Newton's method. We managed to get to level 6 with the twist even condition imposed and to level 5 without it. As in the Siegel gauge, we found several millions of solutions, however, most of them have $|E| \gg 1$, which means that there is only a small probability that they would represent some physical configuration. Subsequently, we took several solutions close to the perturbative vacuum and improved them to higher levels. As in the Siegel gauge, we have found several solutions which are more or less stable in the level truncation scheme, nonetheless only two of them behave sufficiently well to motivate a closer attention. Both solutions are twist even and they appear at level 4.

The properties of the first solution are summarized in table 13. The solution appears to be an analogue of the "double brane" found in [37], but it behaves asymptotically more similarly to the "half brane" solution. The extrapolated values of its energy and $E_{0}$ are non-integers, exhibiting large imaginary parts, so this solution is most likely not physical.

The second solution, which is shown in table 14, behaves slightly better. It is real and the extrapolated values of its energy and the gauge invariant are close to $0 .{ }^{9}$ Therefore, it is possible that this solution is gauge equivalent to the tachyon vacuum. However, the precision of its energy is quite low and the first out-of-gauge equation is not exactly satisfied, so it is possible that this solution is a relict of the level truncation approach as well.

\footnotetext{
${ }^{8}$ The homotopy continuation method is a numerical algorithm used to find all solutions of polynomial equations. See [37, 38] for its application in string field theory and for example [46, 47] for a more general description.

${ }^{9}$ The energy and $E_{0}$ in this appendix are normalized according to the convention adopted in reference [37]. Recall that in the conventions of this work, the analytical value of the vacuum energy and the gauge invariant $E_{0}$ are equal to -1 and 0 respectively.
} 


\begin{tabular}{|c|rll|}
\hline$L$ & Energy & $E_{0}$ & $f_{w}$ \\
\hline 4 & $3.282522-1.78319 i$ & $1.161906-1.69528 i$ & $-0.306633-1.425542 i$ \\
6 & $1.568079-1.64296 i$ & $0.742676-1.23355 i$ & $-0.397242-0.606541 i$ \\
8 & $1.098815-1.33103 i$ & $0.596923-1.08489 i$ & $-0.329192-0.374098 i$ \\
10 & $-0.912291-1.12913 i$ & $0.549851-0.95310 i$ & $-0.279083-0.274678 i$ \\
12 & $-0.819925-0.99526 i$ & $0.512582-0.88831 i$ & $-0.244791-0.221596 i$ \\
14 & $-0.767364-0.90108 i$ & $0.499967-0.82554 i$ & $-0.220437-0.189119 i$ \\
16 & $-0.734482-0.83139 i$ & $0.484460-0.78922 i$ & $-0.202383-0.167350 i$ \\
18 & $-0.712456-0.77768 i$ & $0.480014-0.75187 i$ & $-0.188498-0.151777 i$ \\
20 & $-0.696923-0.73497 i$ & $0.471866-0.72834 i$ & $-0.177490-0.140085 i$ \\
22 & $-0.685523-0.70012 i$ & $0.470194-0.70323 i$ & $-0.168545-0.130974 i$ \\
24 & $-0.676885-0.67110 i$ & $0.465287-0.68656 i$ & $-0.161124-0.123662 i$ \\
\hline$\infty$ & $-0.630-0.318 i$ & $0.456-0.448 i$ & $-0.073-0.048 i$ \\
\hline
\end{tabular}

Table 13. Energy, Ellwood invariant and the first out-of-gauge equation for a "half brane" solution.

\begin{tabular}{|c|rrr|}
\hline$L$ & Energy & $E_{0}$ & $f_{w}$ \\
\hline 4 & -25.8203 & -1.526687 & 0.110970 \\
6 & -11.0589 & -1.027420 & 0.126293 \\
8 & -6.77329 & -0.757402 & -0.021528 \\
10 & -4.89103 & -0.617817 & -0.090068 \\
12 & -3.85610 & -0.524986 & -0.123871 \\
14 & -3.20556 & -0.461964 & -0.142606 \\
16 & -2.75936 & -0.414177 & -0.153968 \\
18 & -2.43407 & -0.377513 & -0.161320 \\
20 & -2.18609 & -0.347829 & -0.166303 \\
22 & -1.99047 & -0.323443 & -0.169790 \\
24 & -1.83196 & -0.302941 & -0.172285 \\
\hline$\infty$ & -0.19 & -0.054 & -0.15 \\
\hline
\end{tabular}

Table 14. Energy, Ellwood invariant and the first out-of-gauge equation for a possible gauge copy of the tachyon vacuum solution. 
Open Access. This article is distributed under the terms of the Creative Commons Attribution License (CC-BY 4.0), which permits any use, distribution and reproduction in any medium, provided the original author(s) and source are credited.

\section{References}

[1] E. Witten, Noncommutative Geometry and String Field Theory, Nucl. Phys. B 268 (1986) 253 [INSPIRE].

[2] M. Schnabl, Analytic solution for tachyon condensation in open string field theory, Adv. Theor. Math. Phys. 10 (2006) 433 [hep-th/0511286] [INSPIRE].

[3] A. Sen, Descent relations among bosonic D-branes, Int. J. Mod. Phys. A 14 (1999) 4061 [hep-th/9902105] [INSPIRE].

[4] A. Sen, Universality of the tachyon potential, JHEP 12 (1999) 027 [hep-th/9911116] [INSPIRE].

[5] Y. Okawa, Comments on Schnabl's analytic solution for tachyon condensation in Witten's open string field theory, JHEP 04 (2006) 055 [hep-th/0603159] [INSPIRE].

[6] Y. Okawa, Analytic methods in open string field theory, Prog. Theor. Phys. 128 (2012) 1001 [INSPIRE].

[7] E. Fuchs and M. Kroyter, Schnabl's $L_{0}$ operator in the continuous basis, JHEP 10 (2006) 067 [hep-th/0605254] [INSPIRE].

[8] E.A. Arroyo, Conservation laws and tachyon potentials in the sliver frame, JHEP 06 (2011) 033 [arXiv: 1103.4830] [INSPIRE].

[9] L. Rastelli and B. Zwiebach, Solving Open String Field Theory with Special Projectors, JHEP 01 (2008) 020 [hep-th/0606131] [INSPIRE].

[10] I. Ellwood and M. Schnabl, Proof of vanishing cohomology at the tachyon vacuum, JHEP 02 (2007) 096 [hep-th/0606142] [INSPIRE].

[11] Y. Okawa, L. Rastelli and B. Zwiebach, Analytic Solutions for Tachyon Condensation with General Projectors, hep-th/0611110 [INSPIRE].

[12] T. Erler, Split String Formalism and the Closed String Vacuum, JHEP 05 (2007) 083 [hep-th/0611200] [INSPIRE].

[13] T. Erler, Split String Formalism and the Closed String Vacuum, II, JHEP 05 (2007) 084 [hep-th/0612050] [INSPIRE].

[14] M. Schnabl, Algebraic Solutions in Open String Field Theory - a Lightning Review, arXiv: 1004.4858 [INSPIRE].

[15] E. Fuchs and M. Kroyter, Analytical Solutions of Open String Field Theory, Phys. Rept. 502 (2011) 89 [arXiv: 0807.4722] [INSPIRE].

[16] M. Kiermaier, A. Sen and B. Zwiebach, Linear b-Gauges for Open String Fields, JHEP 03 (2008) 050 [arXiv:0712.0627] [INSPIRE].

[17] T. Takahashi, Level truncation analysis of exact solutions in open string field theory, JHEP 01 (2008) 001 [arXiv:0710.5358] [INSPIRE].

[18] I. Kishimoto, On numerical solutions in open string field theory, Prog. Theor. Phys. Suppl. 188 (2011) 155 [INSPIRE]. 
[19] E.A. Arroyo, Cubic interaction term for Schnabl's solution using Pade approximants, J. Phys. A 42 (2009) 375402 [arXiv:0905.2014] [InSPIRE].

[20] E. Aldo Arroyo, Level truncation analysis of regularized identity based solutions, JHEP 11 (2011) 079 [arXiv:1109.5354] [INSPIRE].

[21] E.A. Arroyo, Comments on regularization of identity based solutions in string field theory, JHEP 11 (2010) 135 [arXiv:1009.0198] [INSPIRE].

[22] T. Takahashi and S. Tanimoto, Marginal and scalar solutions in cubic open string field theory, JHEP 03 (2002) 033 [hep-th/0202133] [INSPIRE].

[23] T. Erler and M. Schnabl, A Simple Analytic Solution for Tachyon Condensation, JHEP 10 (2009) 066 [arXiv:0906.0979] [INSPIRE].

[24] M. Murata and M. Schnabl, Multibrane Solutions in Open String Field Theory, JHEP 07 (2012) 063 [arXiv:1112.0591] [INSPIRE].

[25] T. Masuda, Comments on new multiple-brane solutions based on Hata-Kojita duality in open string field theory, JHEP 05 (2014) 021 [arXiv: 1211.2649] [INSPIRE].

[26] H. Hata and T. Kojita, Singularities in K-space and Multi-brane Solutions in Cubic String Field Theory, JHEP 02 (2013) 065 [arXiv: 1209.4406] [INSPIRE].

[27] T. Masuda, T. Noumi and D. Takahashi, Constraints on a class of classical solutions in open string field theory, JHEP 10 (2012) 113 [arXiv: 1207.6220] [INSPIRE].

[28] L. Bonora, S. Giaccari and D.D. Tolla, Analytic solutions for Dp-branes in SFT, JHEP 12 (2011) 033 [arXiv:1106.3914] [INSPIRE].

[29] L. Bonora, S. Giaccari and D.D. Tolla, The energy of the analytic lump solution in SFT, JHEP 08 (2011) 158 [Erratum ibid. 04 (2012) 001] [arXiv:1105.5926] [INSPIRE].

[30] M. Kiermaier, Y. Okawa, L. Rastelli and B. Zwiebach, Analytic solutions for marginal deformations in open string field theory, JHEP 01 (2008) 028 [hep-th/0701249] [INSPIRE].

[31] N. Mertes and M. Schnabl, String field representation of the Virasoro algebra, JHEP 12 (2016) 151 [arXiv:1610.00968] [INSPIRE].

[32] M. Jokel, Real Tachyon Vacuum Solution without Square Roots, arXiv:1704.02391 [INSPIRE].

[33] E.A. Arroyo, Comments on real tachyon vacuum solution without square roots, JHEP 01 (2018) 006 [arXiv:1706.00336] [INSPIRE].

[34] N. Moeller and W. Taylor, Level truncation and the tachyon in open bosonic string field theory, Nucl. Phys. B 583 (2000) 105 [hep-th/0002237] [INSPIRE].

[35] D. Gaiotto and L. Rastelli, Experimental string field theory, JHEP 08 (2003) 048 [hep-th/0211012] [INSPIRE].

[36] E.A. Arroyo, A. Fernandes-Silva and R. Szitas, Numerical solution of open string field theory in Schnabl gauge, JHEP 01 (2018) 007 [arXiv:1707.09452] [INSPIRE].

[37] M. Kudrna and M. Schnabl, Universal Solutions in Open String Field Theory, arXiv: 1812.03221 [INSPIRE].

[38] M. Kudrna, Level Truncation Approach to Open String Field Theory, Ph.D. Thesis (2019) https://dspace.cuni.cz/bitstream/handle/20.500.11956/110558/140080054.pdf. 
[39] I. Kishimoto and T. Takahashi, Numerical Evaluation of Gauge Invariants for a-gauge Solutions in Open String Field Theory, Prog. Theor. Phys. 121 (2009) 695 [arXiv:0902.0445] [INSPIRE].

[40] I. Kishimoto and T. Takahashi, Numerical Evaluation of Gauge Invariants for a-gauge Solutions in Open String Field Theory, Theor. Math. Phys. 163 (2010) 710 [arXiv:0910.3025] [INSPIRE].

[41] A. Sen and B. Zwiebach, Tachyon condensation in string field theory, JHEP 03 (2000) 002 [hep-th/9912249] [INSPIRE].

[42] M. Kudrna and C. Maccaferri, BCFT moduli space in level truncation, JHEP 04 (2016) 057 [arXiv: 1601.04046] [INSPIRE].

[43] A. Hashimoto and N. Itzhaki, Observables of string field theory, JHEP 01 (2002) 028 [hep-th/0111092] [INSPIRE].

[44] I. Ellwood, The Closed string tadpole in open string field theory, JHEP 08 (2008) 063 [arXiv:0804.1131] [INSPIRE].

[45] M. Kudrna, C. Maccaferri and M. Schnabl, Boundary State from Ellwood Invariants, JHEP 07 (2013) 033 [arXiv:1207.4785] [INSPIRE].

[46] The Principles of Polynomial Homotopy Continuation Methods, http://homepages.math.uic.edu/ jan/srvart/node4.html.

[47] E.L. Allgower and K. Georg, Introduction Numerical Continuation Methods, Springer-Verlag Berlin Heidelberg (1990). 